\title{
Détermination par traçage isotopique de la valeur fertilisante du phosphate alumino-calci- que : comparaison avec d'autres formes
}

Christian MOREL \& Jean-Claude FARDEAU (*)

COFAZ, Service agronomique, B.P. 133, F 92202 Neuilly-sur-Seine Cedex (*) DB/SRA, CEN Cadarache, F 13108 Saint-Paul-lez-Durance Cedex

Mots clés additionnels : Efficacité relative, coefficient réel d'utilisation, phosphate diammonique, phosphate naturel. phosphate, diammonium phosphate and rock phosphate.

\begin{abstract}
The efficiency of 3 phosphate fertilizers (diammonium phosphate (DAP), aluminium-calcium phosphate and rock phosphate), labelled with $\mathrm{P} 32$, was determined in the greenhouse with Lolium perenne $\mathrm{L}$. The average percent of P utilization of DAP, phospal and rock phosphate, obtained in 5 soils, were respectively $14 \%, 2 \%$ and $0.9 \%$ for a 160-days growth period. These results are expressed in term of relative efficiency by comparison with all the published data concerning dry matter production and $\mathrm{P}$ uptake. The mean value of the relative agronomic efficiency of the phosphal was $38 \%$ in comparison with the water-soluble $P$ form. This relative agronomic efficiency depended on the rate of applied fertilizer, the level of soil phosphorus fertility and the soil reactions with phosphate ions. The data obtained show that the use of labelled phosphate fertilizers allows measurement of the immediate utilization of phosphorus from different fertilizers in all circumstances. These can then be rated, even in soils with high phosphorus level where there is no crop response.
\end{abstract}

Additional key words : Relative agronomic efficiency, percent of phosphorus utilization.

\section{INTRODUCTION}

Au regard des législations, les différentes formes physico-chimiques d'engrais phosphatés sont différenciées, non par le biais de comportements agronomiques mais au moyen de tests de solubilité dans des réactifs conventionnels tels que l'acide citrique, le citrate d'ammonium neutre ou alcalin, les acides minéraux (AFNOR, 1982). De nombreux travaux ont été et continuent d'être conduits pour préciser les conditions d'utilisation les plus rationnelles de chacune des formes. Ils portent, le plus souvent, sur la comparaison de situations les plus opposées, à savoir celles portant d'une part sur les formes solubles eau ou eau citrate 
telles que superphosphate et phosphate d'ammonium et d'autre part sur les formes les plus insolubles eau telles que les phosphates naturels.

L'évolution du prix des engrais phosphatés et plus généralement du coût de la fertilisation par rapport à celui de la production agricole a conduit à voir apparaître ou réapparaître dans certaines parties du monde, et en France en particulier, un certain intérêt pour le phosphate alumino-calcique. Celui-ci est un cas distinct des autres formes d'engrais phosphatés en ce qui concerne ses constituants chimiques, sa solubilité et son traitement industriel. Les références agronomiques sur ces conditions d'utilisation sont beaucoup plus rares que pour les autres formes et pour partie contradictoires principalement lorsque l'on compare les conclusions des études françaises (RADET, 1962 ; GACHON, 1973) à celles des études australiennes (PALMER et al., 1979 ; BOLLAND et al., 1984 ; BOLLAND \& BOWDEN, 1984).

En fait, comme pour tous les autres engrais phosphatés, la difficulté essentielle reste de distinguer au mieux :

- l'effet à court terme sur la culture qui suit l'apport,

- de l'effet résiduel observable sur les cultures, mais seulement par la suite, et qui correspond à la contribution de l'engrais au maintien, ou à l'accroissement, du pool des réserves assimilables du sol.

C'est pourquoi il a semblé intéressant, dans une première phase, de préciser l'effet à court terme de ce type d'engrais. L'objectif retenu a été d'obtenir des données originales portant sur la mesure directe, au moyen du phosphore 32 , du coefficient d'utilisation du phosphore du phosphate alumino-calcique. Cette détermination a été entreprise pour une culture de ray-grass (Lolium perenne, L.), conduite en vase de végétation, dans des sols de $\mathrm{pH}$ compris entre 6,6 et 8,2. Les résultats obtenus ont été comparés à ceux observés, d'une part avec un phosphate naturel, et d'autre part avec un phosphate soluble eau.

\section{MATÉRIEL ET MÉTHODES}

\section{A. Les engrais phosphatés}

Ceux retenus pour cette étude sont au nombre de trois :

- le phosphate diammonique titrant 54 p. 100 de $\mathrm{P}_{2} \mathrm{O}_{5}$ (DAP),

- le phosphate naturel de caroline non calciné (CNC), broyé à $63 \mu$ et titrant 30 p. 100 de $\mathrm{P}_{2} \mathrm{O}_{5}$ et qui représente actuellement le plus assimilable des phosphates naturels exploités (LÉON et al., 1986),

- le phosphate alumino-calcique (tabl. 1). Au moins 2 gisements sont exploités : l'un situé sur l'île de Christmas et l'autre à Thiès (Sénégal). Le minerai de phosphate double de calcium et d'aluminium plus ou moins substitué (FLICOTEAUX, 1982), broyé puis calciné à $550{ }^{\circ} \mathrm{C}$ environ, représente la forme utilisée comme engrais. Il est commercialisé sous le nom de "Calcined Christmas Island C-grade Ore » (CCICP) ou Calciphos s'il vient du premier gisement, et de phospal s'il vient du second.
TABLEAU 1

Composition des différents phosphates alumino-calciques. Chemical contents of different aluminium calcium phosphates.

\begin{tabular}{|c|c|c|c|}
\hline \multirow{2}{*}{$\begin{array}{c}\text { Engrais } \\
\text { Références }\end{array}$} & \multirow{2}{*}{$\begin{array}{c}\text { Phospal } \\
\text { (Sénégal) }\end{array}$} & \multicolumn{2}{|c|}{ CCIP (Christmas) } \\
\hline & & $\begin{array}{c}\text { Mason \& Cox } \\
\text { (1969) }\end{array}$ & $\begin{array}{c}\text { ROBER'TS \& } \\
\text { WHITE (1974) }\end{array}$ \\
\hline $\mathrm{P}_{2} \mathrm{O}_{5} \%$ & 34,6 & 37,8 & 27,9 à 33,4 \\
\hline $\mathrm{CaO} \%$ & 10,9 & & 9,2 à 18,3 \\
\hline $\mathrm{Al}_{2} \mathrm{O}_{3} \%$ & 35,9 & 28,5 & 22,3 à 31,8 \\
\hline $\mathrm{Fe}_{2} \mathrm{O}_{3} \%$ & 9,1 & 11,2 & 10,9 à 19,2 \\
\hline
\end{tabular}

Le marquage, par du phosphore 32 , de ces 3 engrais a été effectué d'une part par synthèse au laboratoire pour le DAP, et d'autre part par activation neutronique pour les 2 autres formes.

La dose choisie pour l'apport a été de 66 p.p.m.P soit l'équivalent de $450 \mathrm{~kg}$ de $\mathrm{P}_{2} \mathrm{O}_{5}$ dans un sol contenant 3000 tonnes de terre/ha. Cette dose qui correspond à une fumure de redressement devait permettre de saisir une partie des mécanismes mis en jeu dans le devenir des engrais phosphatés.

\section{B. Les sols}

Les caractéristiques traditionnelles physicochimiques des 5 sols étudiés, et en particulier leur teneur en $\mathrm{P}$ 《 assimilable » selon 3 méthodes d'extraction chimiques, sont présentées au tableau 2. Au regard des normes françaises d'interprétation du niveau de fertilité phosphorique, seul le sol 2 est insuffisamment pourvu, le sol 1 l'est moyennement et les sols 3, 4 et 5 le seraient suffisamment. Le choix fait sur le $\mathrm{pH}$ des sols, qui s'échelonne de 6,6 à 8,2 , permet d'une part de compléter les informations australiennes ou néozélandaises obtenues pour l'essentiel en sol acide (ROBERTS \& WHITE, 1974 ; PALMER et al., 1979) et d'autre part d'examiner la validité du conseil agronomique préconisant l'emploi du phospal sans restriction en sol acide et neutre et comme fumure de fond en sol calcaire bien pourvu (GROS, 1962).

TABLEAU 2

Caractéristiques physico-chimiques des sols. Physical and chemical characteristics of the soils.

\begin{tabular}{|c|c|c|c|c|c|c|c|}
\hline \multirow[t]{2}{*}{$\mathrm{N}^{\circ}$ sol } & \multirow[t]{2}{*}{ Texture } & \multirow[t]{2}{*}{$\begin{array}{l}\mathrm{pH} \\
\text { eau }\end{array}$} & \multirow[t]{2}{*}{$\mathrm{MO} \%$} & \multirow[t]{2}{*}{$\underset{0 \%}{\mathrm{CaCO}_{3}}$} & \multicolumn{3}{|c|}{$\begin{array}{l}\text { Phosphore extrait } \\
\left(\text { ppm } \mathrm{P}_{2} \mathrm{O}_{5}\right)\end{array}$} \\
\hline & & & & & DYER & $\begin{array}{l}\text { JORET } \\
\text { HEBERT }\end{array}$ & OLSEN \\
\hline 1 & $\begin{array}{l}\text { limono- } \\
\text { sableux }\end{array}$ & 6,6 & 1,8 & 0,2 & 180 & & 71 \\
\hline 2 & limon & 7,2 & 1,4 & 0,1 & 110 & 30 & 39 \\
\hline 3 & limon & 7,4 & 3,0 & 0,7 & 540 & 180 & 209 \\
\hline 4 & limon & 7,6 & 2,1 & 0,2 & & 430 & 152 \\
\hline 5 & calcaire & 8,2 & 2,8 & 67 & & 230 & 99 \\
\hline
\end{tabular}

\section{La conduite des cultures}

Les cultures sont conduites en serre dans des pots en plastique à réserve d'eau, remplis de $1 \mathrm{~kg}$ de sol sec. 
Chaque traitement comporte 5 répétitions. Sur le sol mis en place dans le pot, on a humidifié puis semé $1 \mathrm{~g}$ de graines de Ray grass que l'on a recouvert d'une mince couche de sable grossier destiné à maintenir l'humidité durant la germination. Huit coupes successives, à raison d'une toutes les 3 semaines, ont été réalisées. La végétation a été entretenue en apportant, après chaque coupe, 60 p.p.m.N sous forme de nitrate de potassium.

\section{Déterminations réalisées}

- La matière sèche produite est déterminée après séchage à l'étuve à $60^{\circ} \mathrm{C}$.

- Le phosphore total exporté est obtenu par dosage colorimétrique après calcination de la matière sèche puis reprise des cendres en milieu acide.

- Le phosphore 32 est déterminé par effet Cerenkov pour éliminer la contribution, dans les engrais irradiés et dans les récoltes ayant poussé en leur présence, des radioisotopes autres que le ${ }^{32} \mathrm{P}$ et en particulier le ${ }^{45} \mathrm{Ca}$.

\section{E. Traitements des données. Expression des résultats}

Il n'existe pas actuellement de définition, et par voie de conséquence de méthode de détermination, de la valeur absolue de l'assimilabilité d'un élément contenu dans un engrais (BLACK \& SCOTT, 1956 ; WHITE et al., 1956) ; par contre on peut toujours définir une valeur relative, appelée efficacité relative (ER), d'un élément nutritif d'un constituant, par rapport au même élément présent dans un autre constituant pris comme référence, en comparant l'effet des 2 constituants sur une variable biologique ou physico-chimique du système. Cette variable peut être, soit le rendement en matière sèche brut ou commercialisable, soit la quantité de l'élément prélevé, soit la quantité de l'élément présente dans le sol sous une forme jugée assimilable. Selon la référence choisie et la méthode de comparaison retenue, on a abouti à plusieurs appréciations de l'efficacité relative. Cet article est l'occasion de les présenter, puis de les utiliser et enfin de les commenter au vu des données expérimentales. Il s'agit, en désignant par X la variable étudiée, de calculer :

$\mathrm{ER}_{1}($ p. 100) $=\mathrm{X}($ sol + engrais $) / \mathrm{X}($ sol témoin $) \times 100$.

Ce traitement des données est de loin le plus fréquent. La référence est la réponse agronomique du sol témoin. Une telle approche élimine pratiquement toute comparaison d'efficacité relative de divers engrais placés dans plusieurs sols.

On calcule aussi :

$\mathrm{ER}_{2}($ p. 100) $=[\mathrm{X}($ sol $+\mathrm{ET}$ dose 2$)-\mathrm{X}($ sol $+\mathrm{ET}$ dose 1$)]$

$[\mathrm{X}($ sol $+\mathrm{EE}$ dose 2$)-\mathrm{X}($ sol + EE dose 1$)] \times 100$

avec, ET désignant l'engrais testé par rapport à l'engrais étalon, EE.

Cette démarche a donné lieu à de nombreuses variantes pour l'estimation de l'efficacité relative. La formule (2) peut prendre la forme suivante :
Cette présentation montre que $\mathrm{ER}_{2}$ peut être calculée, de manière très générale, au moyen des courbes de réponses aux engrais (testés et étalons), et correspond alors dans l'intervalle (dose 2 - dose 1), aux rapports des pentes des courbes représentatives de la variable $X$ en fonction des doses d'engrais. Partant de là, certains (PALMER et al., 1979) ont estimé qu'en raison des variations de la valeur de $\mathrm{ER}_{2}$ en fonction des valeurs choisies pour les doses 1 et 2 , il paraissait souhaitable de ne prendre en considération que la valeur de $E_{2}$ lorsque la dose 2 tend vers la valeur de la dose 1 qui elle-même tendrait vers zéro. On est donc en droit d'admettre que ces diverses approches ne sont pas conceptuellement différentes et, qu'en conséquence tous les résultats ainsi obtenus peuvent être comparés entre eux. Cependant la difficulté matérielle que représente sur le terrain la mise en place d'essais courbes de réponse avec plusieurs formes d'engrais phosphatés a conduit de très nombreux expérimentateurs à n'utiliser qu'une dose non nulle de phosphore. La formule (2) donne alors naissance à la valeur $\mathrm{ER}_{3}$ :

$$
\begin{aligned}
E_{3}(p .100)= & {[X(\text { sol }+E T)-X(\text { sol témoin })] } \\
& \quad[X(\text { sol }+E E)-X(\text { sol témoin })] \times 100 .
\end{aligned}
$$

Lorsque la variable analysée est le prélèvement de phosphore, $\mathrm{ER}_{3}$ n'est rien d'autre que le rapport des coefficients d'utilisation apparent $\mathrm{du}$ phosphore apporté par les 2 engrais. Il est au moins un cas où, par définition, une telle mesure est vouée à l'échec. Il s'agit de la situation où le sol est à un niveau supérieur à celui dit d'entretien (LoUÉ, 1982). Dans une telle situation seul le traçage isotopique, au moyen de ${ }^{32} \mathrm{P}$ ou de ${ }^{33} \mathrm{P}$, du phosphore présent dans les différents engrais permet de connaître non plus le coefficient apparent d'utilisation mais le coefficient réel d'utilisation du phosphore (CRU-P) des différents engrais. C'est pourquoi on propose une estimation supplémentaire de l'efficacité relative utilisable quelle que soit la richesse du sol :

$\mathrm{ER}_{4}($ p. 100) $=[\mathrm{CRU}-\mathrm{P}$ (engrais testé) $/$

CRU-P (engrais étalon) $] \times 100$.

Il faut noter que de toutes les efficacités relatives précédemment définies, $\mathrm{ER}_{4}$ est la seule qui n'utilise comme référence qu'un engrais et non pas un mélange sol-engrais. Nous pensons qu'il s'agit là d'un progrès quand l'objectif est précisément de caractériser des engrais les uns par rapport aux autres.

Dans la mesure où, en ce qui concerne le phosphate alumino-calcique, il n'existe aucune référence bibliographique sur la valeur du coefficient réel d'utilisation du phosphore qu'il contient, les résultats présentés complèteront de manière significative les données plus anciennes portant sur le rendement matière sèche ou sur le prélèvement de phosphore.

\section{RÉSULTATS ET DISCUSSION}

\section{A. Données culturales}

Les résultats cumulés des 8 coupes successives sont présentés dans le tableau 3. L'objectif du travail étant

$\mathrm{ER}_{2}($ p. 100$)=\frac{[\mathrm{X}(\text { sol }+\mathrm{ET} \text { dose } 2)-\mathrm{X}(\text { sol }+\mathrm{ET} \text { dose } 1)] /(\text { dose } 2-\text { dose } 1)}{[\mathrm{X}(\text { sol }+\mathrm{EE} \text { dose } 2)-\mathrm{X}(\text { sol }+ \text { EE dose } 1)] /(\text { dose } 2-\text { dose } 1)} \times 100$. 
TABLEAU 3

Résultats culturaux cumulés sur les 8 coupes. Pour chaque sol, des chiffres suivis de la même lettre ne diffèrent pas significativement au seuil de 5 p. 100.

Dry matter yield, $P$ uptake and percent of $P$ utilization for the 8 cuts (160 days growth). For each soil, numbers followed by the same letter did not differ significantly at the $5 \%$ level.

\begin{tabular}{|c|c|c|c|c|c|c|c|c|c|c|c|}
\hline \multirow[b]{2}{*}{$\begin{array}{c}\text { Engrais } \\
\text { sols }\end{array}$} & \multicolumn{4}{|c|}{ Matière sèche (MS) g/kgsol } & \multicolumn{4}{|c|}{ Exportation de $\mathrm{P}(\mathrm{Qp}) \mathrm{mgP} / \mathrm{kgsol}$} & \multicolumn{3}{|c|}{ CRU-P $(\%)$} \\
\hline & Témoin & DAP & Phospal & $\mathrm{CNC}$ & Témoin & DAP & Phospal & $\mathrm{CNC}$ & DAP & Phospal & $\mathrm{CNC}$ \\
\hline $1 \mathrm{pH}=6,8$ & $8,0 \mathrm{a}$ & $8,8 \mathrm{a}$ & $8,8 \mathrm{a}$ & $9,3 \mathrm{a}$ & $39,0 \mathrm{a}$ & $47,7 b$ & $43,1 \mathrm{a}$ & $45,3 \mathrm{a}$ & $16,1 \mathrm{a}$ & $2,0 \mathrm{~b}$ & $1,7 \mathrm{~b}$ \\
\hline $2 \mathrm{pH}=7,2$ & $5,7 \mathrm{a}$ & $8,0 \mathrm{~b}$ & $6,4 \mathrm{c}$ & 6,0 a c & $16,2 \mathrm{a}$ & $29,6 \mathrm{~b}$ & $20,1 \mathrm{a}$ & $17,8 \mathrm{a}$ & $15,3 \mathrm{a}$ & $2,0 \mathrm{~b}$ & $1,0 \mathrm{c}$ \\
\hline $3 \mathrm{pH}=7,4$ & $6,3 \mathrm{a}$ & $6,1 \mathrm{a}$ & $6,0 \mathrm{a}$ & $6,6 \mathrm{a}$ & $40,2 \mathrm{a}$ & $35,2 \mathrm{a}$ & $37,4 \mathrm{a}$ & $41,9 \mathrm{a}$ & $7,8 \mathrm{a}$ & $1,2 \mathrm{~b}$ & $0,4 \mathrm{c}$ \\
\hline $4 \mathrm{pH}=7,6$ & $9,6 a$ & 9,4 a & 9,3 a & $10,4 a$ & $46,9 \mathrm{a}$ & $55,1 \mathrm{~b}$ & $50,3 \mathrm{~b}$ & $52,3 \mathrm{~b}$ & $15,5 \mathrm{a}$ & $2,1 \mathrm{~b}$ & $0,9 \mathrm{c}$ \\
\hline $5 \mathrm{pH}=8,2$ & $6,1 \mathrm{a}$ & $6,3 \mathrm{a}$ & $6,6 \mathrm{a}$ & 6,5 a & $29,3 \mathrm{a}$ & $37,2 \mathrm{~b}$ & $34,8 c$ & $32,0 \mathrm{a}$ & 14,1 a & $2,6 \mathrm{~b}$ & $0,5 \mathrm{c}$ \\
\hline
\end{tabular}

de comparer différents engrais dans un sol et non le comportement des différents sols vis-à-vis des divers engrais, l'analyse statistique n'a porté que sur les différents traitements dans un sol donné. Des lettres identiques pour un sol donné et une variable, dans les divers tableaux, indiquent que les résultats ne sont pas significativement différents au seuil de 5 p. 100. L'analyse de ces données conduit aux remarques suivartes:

- La quantité de matière sèche récoltée sur les sols $1,3,4$ et 5 n'a pas été modifiée par l'apport de phosphore, quelle que soit sa forme. Le niveau élevé de fertilité phosphorique, apprécié par les méthodes chimiques classiques d'extraction, suffit à expliquer ce résultat. C'est uniquement dans le sol 2 , de teneur plus faible, que le DAP a significativement accru les rendements en matière sèche. Un tel résultat obtenu en vases de végétation est conforme aux observations de plein champ.

- A l'exception du sol 3, les exportations cumulées de phosphore sont, par rapport au témoin sans $\mathbf{P}$, plus élevées dans tous les sols en présence de DAP mais ne sont pas différenciées en présence de phospal ou de phosphate naturel. C'est dire que dans ces cas le coefficient d'utilisation apparent du phosphore n'est pas significativement différent de zéro.

- Le coefficient réel d'utilisation du phosphore des différents engrais, par la culture de Ray grass, n'est cependant pas nul. Le CRU-P du DAP est compris entre 7,8 p. 100 et 16,1 p. 100 , celui des 2 autres formes entre 0,4 p. 100 et 2,6 p. 100 . Celui du phospal est dans les 5 sols étudiés, supérieur à celui du phosphate de Caroline. Ces dernières valeurs sont si faibles qu'elles ne peuvent entraîner ni une augmentation de rendement ni un accroissement de prélèvement de phosphore ce qui est en accord avec la détermination du coefficient apparent.

L'analyse est par contre différente dans le cas du DAP pour les sols $1,3,4$ et 5 . Ici la contribution du phosphore de l'engrais est suffisamment importante pour provoquer un accroissement de la production de matière sèche et du prélèvement de phosphore si cet élément avait été un facteur limitant de la production. Ce n'était pas le cas et l'on a simplement assisté, au niveau de la nutrition phosphatée des cultures, à une substitution du phosphore des réserves du sol par celui originaire de l'engrais. On est donc en droit de conclure que malgré une apparente identité de comporte- ment des 3 formes d'engrais utilisés l'un d'eux est effectivement utilisable dans ces sols alors que les 2 autres ne le sont pas.

- L'écart entre les CRU-P des formes insolubleseau crô̂t avec le pH (fig. 1). Ce résultat n'a pas pour origine un accroissement de l'utilisation du phosphore du phospal mais une diminution de celle du phosphate de Caroline qui passe de 1,7 p. 100 à $\mathrm{pH}=6,6$, à 0,5 p. 100 à $\mathrm{pH}=8,2$.

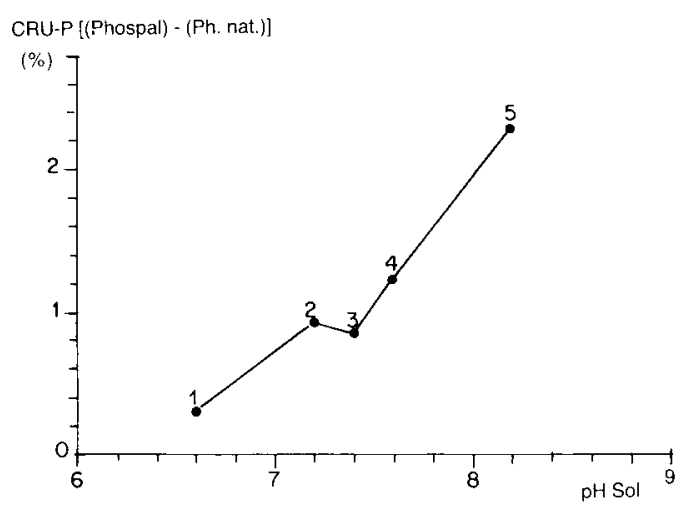

Figure 1

Différence entre le CRU-P du phospal et du phosphate de Caroline en fonction du $\mathrm{pH}$.

Difference between percent of utilization of phospal and Caroline rock phosphate versus soil $\mathrm{pH}$.

\section{B. Efficacité relative à court terme des divers engrais}

Les calculs de $\mathrm{ER}_{3}$ et de $\mathrm{ER}_{4}$ dépendent de l'engrais étalon choisi. Nos résultats sont présentés en faisant appel aux 2 formes extrêmes : le DAP et le phosphate naturel. En effet le choix de la forme soluble-eau est de loin le plus fréquent, la majorité des agronomes admettant implicitement leur supériorité, mais il arrive parfois que la forme la plus insoluble soit choisie comme référence (FRIED \& DEAN, 1952).

\section{Analyse des résultats}

Les valeurs calculées de $\mathrm{ER}_{1}, \mathrm{ER}_{3}$ et $\mathrm{ER}_{4}$ sont groupées au tableau 4. Le symbole "IND » correspond à une valeur indéterminée ayant pour origine le fait que 
TABLEAU 4

Efficacités relatives agronomiques. Deux lettres identiques indiquent des résultats non significativement différents au seuil de 5 p. 100. Relative agronomic efficiency. Two different letters mean significant difference at the $5 \%$ level.

\begin{tabular}{|c|c|c|c|c|c|c|c|c|c|}
\hline \multirow[t]{3}{*}{$\mathrm{N}^{\circ}$ sol } & \multirow[t]{3}{*}{ Engrais } & \multicolumn{2}{|c|}{$\mathrm{ER}_{1}(\%)$} & \multicolumn{4}{|c|}{$\mathrm{ER}_{3}(\mathbf{\%})$} & \multicolumn{2}{|c|}{$\mathrm{ER}_{4}(\boldsymbol{0})$} \\
\hline & & \multirow[b]{2}{*}{ MS } & \multirow[b]{2}{*}{ Qp } & \multicolumn{2}{|c|}{$\begin{array}{l}\text { DAP } \\
\text { étalon }\end{array}$} & \multicolumn{2}{|c|}{$\begin{array}{c}\mathrm{CNC} \\
\text { étalon }\end{array}$} & \multirow[t]{2}{*}{$\begin{array}{c}\text { DAP } \\
\text { étalon }\end{array}$} & \multirow[t]{2}{*}{$\begin{array}{c}\text { CNC } \\
\text { étalon }\end{array}$} \\
\hline & & & & MS & Qp & MS & Qp & & \\
\hline 1 & $\begin{array}{c}\text { DAP } \\
\text { Phospal } \\
\text { CNC }\end{array}$ & $\begin{array}{l}110 \mathrm{a} \\
110 \mathrm{a} \\
122 \mathrm{a}\end{array}$ & $\begin{array}{l}122 \mathrm{a} \\
110 \mathrm{~b} \\
116 \mathrm{~b}\end{array}$ & $\begin{array}{l}100 \mathrm{a} \\
100 \mathrm{a} \\
162 \mathrm{a}\end{array}$ & $\begin{array}{r}100 \mathrm{a} \\
47 \mathrm{~b} \\
72 \mathrm{~b}\end{array}$ & $\begin{array}{r}61 \mathrm{a} \\
61 \mathrm{a} \\
100 \mathrm{a}\end{array}$ & $\begin{array}{r}138 \mathrm{a} \\
65 \mathrm{a} \\
100 \mathrm{a}\end{array}$ & $\begin{array}{r}100 \mathrm{a} \\
12 \mathrm{~b} \\
10 \mathrm{c}\end{array}$ & $\begin{array}{l}947 \mathrm{a} \\
118 \mathrm{~b} \\
100 \mathrm{~b}\end{array}$ \\
\hline 2 & $\begin{array}{c}\text { DAP } \\
\text { Phospal } \\
\text { CNC }\end{array}$ & $\begin{array}{l}140 \mathrm{a} \\
112 \mathrm{~b} \\
105 \mathrm{~b}\end{array}$ & $\begin{array}{l}182 a \\
124 b \\
110 b\end{array}$ & $\begin{array}{r}100 \mathrm{a} \\
30 \mathrm{~b} \\
13 \mathrm{~b}\end{array}$ & $\begin{array}{r}100 \mathrm{a} \\
29 \mathrm{~b} \\
12 \mathrm{~b}\end{array}$ & $\begin{array}{l}766 \mathrm{a} \\
233 \mathrm{~b} \\
100 \mathrm{~b}\end{array}$ & $\begin{array}{l}837 \mathrm{a} \\
243 \mathrm{~b} \\
100 \mathrm{~b}\end{array}$ & $\begin{array}{r}100 a \\
13 b \\
7 c\end{array}$ & $\begin{array}{l}1530 \mathrm{a} \\
200 \mathrm{~b} \\
100 \mathrm{c}\end{array}$ \\
\hline 3 & $\begin{array}{c}\text { DAP } \\
\text { Phospal } \\
\text { CNC }\end{array}$ & $\begin{array}{r}97 \mathrm{a} \\
95 \mathrm{a} \\
104 \mathrm{a}\end{array}$ & $\begin{array}{r}97 \mathrm{a} \\
93 \mathrm{~b} \\
103 \mathrm{a}\end{array}$ & $\begin{array}{l}\text { IND } \\
\text { IND } \\
\text { IND }\end{array}$ & $\begin{array}{l}\text { IND } \\
\text { IND } \\
\text { IND }\end{array}$ & $\begin{array}{l}\text { IND } \\
\text { IND } \\
\text { IND }\end{array}$ & $\begin{array}{l}\text { IND } \\
\text { IND } \\
\text { IND }\end{array}$ & $\begin{array}{r}100 \mathrm{a} \\
15 \mathrm{~b} \\
5 \mathrm{c}\end{array}$ & $\begin{array}{r}1950 \mathrm{a} \\
300 \mathrm{~b} \\
100 \mathrm{c}\end{array}$ \\
\hline 4 & $\begin{array}{c}\text { DAP } \\
\text { Phospal } \\
\text { CNC }\end{array}$ & $\begin{array}{r}98 \mathrm{a} \\
97 \mathrm{a} \\
108 \mathrm{a}\end{array}$ & $\begin{array}{l}117 \mathrm{a} \\
107 \mathrm{~b} \\
111 \mathrm{~b}\end{array}$ & $\begin{array}{l}\text { IND } \\
\text { IND } \\
\text { IND }\end{array}$ & $\begin{array}{r}100 \mathrm{a} \\
41 \mathrm{~b} \\
66 \mathrm{~b}\end{array}$ & $\begin{array}{l}\text { IND } \\
\text { IND } \\
\text { IND }\end{array}$ & $\begin{array}{r}151 \mathrm{a} \\
63 \mathrm{~b} \\
100 \mathrm{~b}\end{array}$ & $\begin{array}{r}100 \mathrm{a} \\
14 \mathrm{~b} \\
6 \mathrm{c}\end{array}$ & $\begin{array}{r}1722 \mathrm{a} \\
233 \mathrm{~b} \\
100 \mathrm{c}\end{array}$ \\
\hline 5 & $\begin{array}{c}\text { DAP } \\
\text { Phospal } \\
\text { CNC }\end{array}$ & $\begin{array}{l}103 \mathrm{a} \\
108 \mathrm{a} \\
105 \mathrm{a}\end{array}$ & $\begin{array}{l}127 \mathrm{a} \\
118 \mathrm{~b} \\
109 \mathrm{~b}\end{array}$ & $\begin{array}{l}100 \mathrm{a} \\
250 \mathrm{a} \\
100 \mathrm{a}\end{array}$ & $\begin{array}{r}100 \mathrm{a} \\
69 \mathrm{~b} \\
34 \mathrm{~b}\end{array}$ & $\begin{array}{r}50 \mathrm{a} \\
125 \mathrm{a} \\
100 \mathrm{a}\end{array}$ & $\begin{array}{l}292 \mathrm{a} \\
203 \mathrm{~b} \\
100 \mathrm{~b}\end{array}$ & $\begin{array}{r}100 \mathrm{a} \\
20 \mathrm{~b} \\
4 \mathrm{c}\end{array}$ & $\begin{array}{r}2820 \mathrm{a} \\
560 \mathrm{~b} \\
100 \mathrm{c}\end{array}$ \\
\hline
\end{tabular}

la récolte, dans les traitements fertilisés, a été inférieure, mais non significativement différente, à celle des pots témoins. Dans tous les autres cas on a calculé les valeurs de $\mathrm{ER}_{1}, \mathrm{ER}_{3}$ et $\mathrm{ER}_{4}$. Les différences significatives apparaissent dans le tableau 4 sous forme de lettres différentes. Ces données conduisent aux commentaires suivants :

- Les valeurs très différentes, pour un traitement donné, de $\mathrm{ER}_{1}, \mathrm{ER}_{3}$ et $\mathrm{ER}_{4}$ montrent l'importance, d'une part du choix du critère agronomique utilisé pour apprécier la réponse culturale (rendement, prélèvement), et d'autre part de la méthode de comparaison choisie (sol témoin servant de référence, engrais étalon, etc...). Ce constat permet d'imaginer que le choix d'un auteur ou d'un expérimentateur n'est pas forcément innocent et, parfois, pourrait être fonction du résultat espéré. Cette seule situation suffirait à expliquer la grande diversité des conclusions relatives au choix des engrais phosphatés employés. Il faut aussi insister sur le fait que des valeurs apparemment très éloignées de $\mathrm{ER}_{3}$ ne sont pas obligatoirement significativement différentes. La détermination d'un tel indice repose sur le calcul d'une différence de rendement pour lequel l'erreur statistique est d'autant plus grande que le rendement en présence de l'étalon est voisin de celui de l'engrais testé. Il en résulte que l'emploi d'un tel indice est très délicat en l'absence de traitement statistique.

- Les variations relatives de $E_{1}$ et de $E_{3}$ sont plus faibles pour la matière sèche que pour le prélèvement global de phosphore. On retrouve là le fait que la richesse d'un sol en phosphore assimilable est loin d'être l'unique facteur contrôlant la production de matière sèche.

- Les indices $\mathrm{ER}_{1}$ et $\mathrm{ER}_{3}$ ne permettent pas de proposer un classement unique des différents engrais étudiés. Ce résultat n'est d'ailleurs pas surprenant dans la mesure où les références retenues pour les calculs sont soit un sol, soit la différence du comportement du sol, avec et sans engrais.
- Le critère $E R_{4}$, qui fait référence, non pas au phosphore total prélevé mais à celui réellement originaire des engrais, permet de proposer un classement indépendant des sols étudiés. En effet, les calculs effectués en prenant le DAP comme étalon montrent que l'efficacité relative du phosphore alumino-calcique est supérieure à celle du phosphate naturel et les calculs conduits avec le phosphate naturel comme étalon montre que le DAP est supérieur au phospal.

- Les valeurs de $\mathrm{ER}_{3}$ sont supérieures à celles de $\mathrm{ER}_{4}$ lorsque l'engrais de référence est le DAP. En d'autres termes le rapport des coefficients apparents du phosphore diffère significativement du rapport des coefficients réels d'utilisation. Les calculs montrent que le supplément, par rapport au témoin sans $P$, de phosphore prélevé dans les traitements où l'on a apporté des formes insolubles-eau ne provient pas en totalité de l'engrais, mais a pour partie le sol pour origine. Dans ces conditions on est conduit à estimer, que dans ces sols, ces engrais, en provoquant une exploration racinaire supplémentaire $\mathrm{du}$ phosphore $\mathrm{du}$ sol, agissent non du fait du phosphore qu'ils contiennent mais en constituant un facteur favorable au développement racinaire.

\section{Discussion, comparaison des résultats et des don- nées bibliographiques}

\section{a) Absence de réponse culturale}

Dans de très nombreuses expériences au champ ou en pots de cultures (cas des sols 1, 3, 4 et 5 de notre expérience), l'apport d'engrais phosphatés n'entraîne pas de modification de la production de matière sèche. Ces résultats, quoique les plus nombreux, ne sont que rarement publiés et l'on peut estimer que seulement 10 p. 100 d'entre eux environ font l'objet de publications. Il en résulte que ceux où apparaît le phosphate alumino-calcique sont relativement rares (GAROLA, 1956 ; BURON, 1965 ; LEFÈVRE \& HiROUX, 1965). 
L'analyse en terme de $\mathrm{ER}_{1}$ de tels résultats aboutit à une valeur de $\mathrm{ER}_{1}=100$ p. 100 quel que soit l'engrais. Certains agronomes en déduisent qu'il y a une équivalence entre le phospal et la forme soluble eau (SINGH, 1983). Cette interprétation ne paraît pas justifiée. En effet un indice tel que $\mathrm{ER}_{3}$ prend alors la valeur indéterminée $0 / 0$ pour tous les traitements, c'est-à-dire qu'elle interdit toute conclusion en matière de comparaison d'engrais. On ne devrait alors conclure qu'à l'inefficacité des engrais dans les conditions culturales où ils ont été testés. Cette absence de réponse culturale est expliquée par une très faible utilisation des engrais par les cultures due soit à l'existence d'un très fort pouvoir fixateur du sol (COUEY et al., 1968), soit à un niveau élevé de fertilité phosphorique des sols (COOKE, 1967 ; BONIFACE et al., 1975 ; GACHON, 1977). En fait, les résultats que nous avons présentés montrent que, dans les sols riches en phosphore assimilable, cette interprétation est erronée dans la mesure où les coefficients d'utilisation du DAP sont loin d'être négligeables et qu'en fait on assiste à un transfert d'alimentation phosphatée du sol vers l'engrais. Seul le critère ER $_{4}$ permet de renseigner sur les mécanismes en jeu et de porter un jugement de valeur sur la participation des différents engrais à la nutrition des cultures.

\section{b) Existence d'une réponse culturale}

Nous avons recensé une vingtaine de publications comportant des résultats culturaux où une réponse agronomique significative au phosphate aluminocalcique a été obtenue. En raison de la diversité des informations bibliographiques, le parti retenu a été d'utiliser une partie des données pour calculer la valeur de $\mathrm{ER}_{3}$ pour la variable matière sèche, l'étalon étant le superphosphate. Cette approche concerne une dizaine de publications (Tendille, 1954 ; MALTERRE \& FIORAMONTI, 1957 ; RADET, 1958 ; MORICEAU \& TINTIGNAC, 1963 ; COPPENET \& DUVAL, 1965 ; HEBERT \& RÉMY, 1965 ; MASON \& COX, 1969 ; LIPSETT \& WILLIAMS, 1970 ; ROBERTS \& WHITE, 1974 ; TRUONG BiNH et al., 1984 ; Bolland et al., 1986). Il a paru également intéressant de prendre en compte les valeurs de $\mathrm{ER}_{2}$, déduites non plus d'un apport unique mais des courbes de réponses établies en présence de plusieurs doses d'engrais phosphatés (TEITZEL \& BRUCE, 1969 ; MASON \& COX, 1969 ; LIPSETT \& WILLIAMS, 1970 ; Muller, 1970 ; PALMER et al., 1979 ; BOLLAND \& BOWDEN, 1982 ; BOLLAND et al., 1984 ; BOLLAND \& BowDEN, 1984). Dans la mesure où nous avons préalablement montré qu'il n'existait pas de différences conceptuelles entre $\mathrm{ER}_{2}$ et $\mathrm{ER}_{3}$ nous avons estimé qu'il était possible de calculer une valeur moyenne de cet ensemble de données bibliographiques. Cette valeur moyenne d'efficacité relative du phosphate aluminocalcique par rapport au superphosphate s'établit à 38,5 p. 100 , son écart type étant de 27 p. 100 . On est donc en droit d'admettre que dans les situations agronomiques où s'observera une réponse annuelle significative à l'addition d'engrais phosphaté celle du phosphate alumino-calcique ne sera que de 38 p. 100 de celle obtenue avec les formes solubles.

Cette valeur est du même ordre de grandeur que celle obtenue dans le sol 2, le seul où le phosphal procure un effet matière sèche. Cette valeur est cependant légère- ment supérieure à la valeur moyenne de $\mathrm{ER}_{4}=16 \mathrm{p} .100$ mais celle-ci correspond pour 4 sols sur 5 à des traitements ne faisant pas apparaître de réponse agronomique significative.

L'analyse bibliographique des facteurs susceptibles d'influer sur la valeur de l'efficacité relative permet d'expliquer cette différence dans la mesure où interviennent :

- La dose d'engrais choisie pour réaliser les déterminations. Comme l'ont montré PALMER et al. (1979), à partir de modèles liant le rendement et la dose, l'efficacité relative diminue quand les apports croissent.

- Le niveau de fertilité phosphorique des sols. Les écarts de rendement entre les parcelles témoins et les parcelles fertilisées diminuent lorsque les sols s'enrichissent en forme assimilable. Il en résulte que les estimations de $\mathrm{ER}_{3}$ basées sur le calcul d'une différence deviennent illusoires. Tel est le cas dans nos propres expériences pour les sols 3, 4 et 5 ainsi que dans des essais au champ lorsque les sols ont été largement engraissés (COLLIER \& GACHON, 1965 ; GACHON, 1965 ; HEBERT, 1965 ; HEBERT \& RÉMY, 1965).

- L'importance de la réaction des sols avec les ions phosphate. Ce phénomène concerne prioritairement les formes solubles dans l'eau. L'activité des ions phosphate d'un apport de phosphate soluble-eau décroît rapidement dans les toutes premières semaines qui suivent l'addition de phosphore au sol. Le fait, mis en évidence de longue date (BARBIER \& TYSKIEWICZ, 1952 ; BLANCHET et al., 1971 ; ARVIEU, 1980) est également observable lors des études culturales (JAHIEL \& MOREL, 1983 ; FARDEAU et al., 1984). Un tel mécanisme touche beaucoup moins des formes insolubes-eau ; le résultat est une diminution, plus rapide au cours du temps, de l'efficacité des formes solubles-eau que des formes insolubles-eau. Il en résultera un accroissement des paramètres tels que $E_{3}$ et $\mathrm{ER}_{4}$ qu'aucune mesure n'autorise à interpréter comme une augmentation de la valeur fertilisante des formes insolubles. Cette remarque suffit à expliquer aussi bien les résultats publiés par DEPARDON \& BURON (1953, $1954,1955,1956,1957$ et 1958), par RADET (1958) où les apports ont été réalisés plusieurs mois avant la culture, que les résultats présentés par TRUONG BINH

TABLEAU 5

Efficacité agronomique relative $\left(E R_{4}\right)$ à court terme du phospal et d'un phosphate naturel.

Relative agronomic efficiency ( $\left.E R_{4}\right)$ for phospal and for a rock phosphate.

\begin{tabular}{|c|c|c|c|c|c|c|}
\hline \multicolumn{2}{|l|}{$\mathrm{N}^{\circ} \mathrm{sol}$} & 1 & 2 & 3 & 4 & 5 \\
\hline \multicolumn{7}{|c|}{$\begin{array}{l}\text { Cumul des engrais } \\
\text { Coupes }\end{array}$} \\
\hline 1 à 8 & $\begin{array}{l}\text { Phospal } \\
\text { CNC }\end{array}$ & $\begin{array}{l}12 \\
10\end{array}$ & $\begin{array}{r}13 \\
7\end{array}$ & $\begin{array}{r}15 \\
5\end{array}$ & $\begin{array}{r}14 \\
6\end{array}$ & $\begin{array}{r}20 \\
4\end{array}$ \\
\hline 1 à 4 & $\begin{array}{l}\text { Phospal } \\
\text { CNC }\end{array}$ & $\begin{array}{r}10 \\
9\end{array}$ & $\begin{array}{r}14 \\
7\end{array}$ & $\begin{array}{r}11 \\
5\end{array}$ & $\begin{array}{r}13 \\
5\end{array}$ & $\begin{array}{r}19 \\
3\end{array}$ \\
\hline 5 à 8 & $\begin{array}{l}\text { Phospal } \\
\text { CNC }\end{array}$ & $\begin{array}{l}16 \\
13\end{array}$ & $\begin{array}{r}12 \\
6\end{array}$ & $\begin{array}{r}29 \\
5\end{array}$ & $\begin{array}{r}19 \\
9\end{array}$ & $\begin{array}{r}25 \\
4\end{array}$ \\
\hline
\end{tabular}


et al. (1984) où le coefficient d'utilisation apparent du DAP ne dépassait pas 3 p. 100. Ce type d'interprétation peut également s'appliquer à nos données. En effet dans le tableau 5 nous avons indiqué les valeurs de $E_{4}$ cumulées pour les coupes 1 à 4 et 5 à 8 ; dans 4 des 5 sols $E_{4}$ croît de la première période à la seconde. La figure 2 représente l'évolution, dans les 5 sols étudiés, du coefficient d'utilisation réel cumulé du phosphore du DAP. Il résulte de la comparaison des données du tableau 5 et du graphique 2 que l'augmentation de $E_{4}$ au cours du temps ne doit pas être interprétée comme un accroissement de la disponibilité du phospal mais comme une diminution de l'utilisation du phosphore du DAP, par rapport à sa valeur initiale. Le même commentaire s'applique également au phosphate naturel.

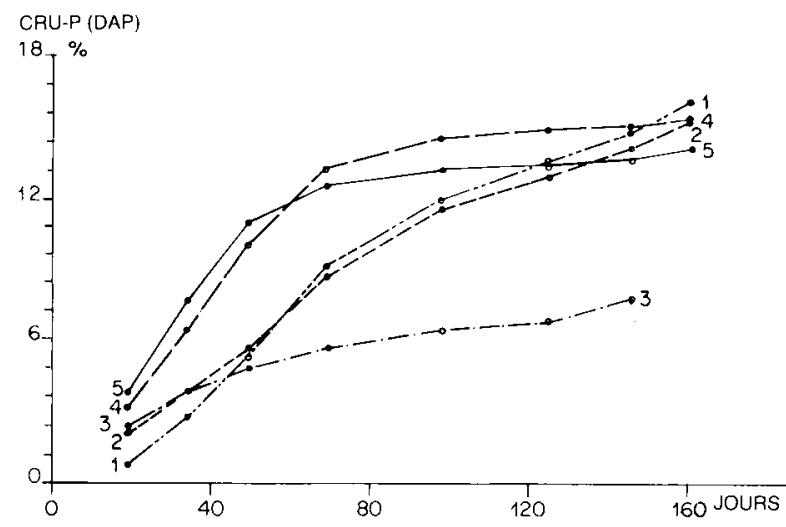

Figure 2

Coefficient d'utilisation cumulé du phosphore du DAP

Cumulated percent of $P$ utilization of DAP.

\section{CONCLUSION}

L'effet à court terme, c'est-à-dire sur la culture qui suit immédiatement l'enfouissement d'engrais du phosphate d'ammonium, du phospal et d'un phosphate naturel originaire de Caroline (USA), a été analysé au moyen de 3 critères d'efficacité relative portant sur 2 paramètres : la matière sèche et le prélèvement de phosphore. Dans les 5 sols expérimentés qui, à l'exception d'un seul, étaient largement pourvus en $\mathrm{P}$ assimilable, l'apport des engrais à la dose de 66 p.p.m.P n'a pas provoqué d'accroissement significatif de la production de matière sèche par rapport au traitement non fertilisé. L'analyse des données montre qu'en pareille circonstance il serait plus juste de parler d'inefficacité agronomique des différents engrais que d'identité de comportement. En effet l'utilisation du traçage isotopique par le ${ }^{32} \mathrm{P}$ du phosphore des engrais démontre, que malgré l'absence de réponse agronomique, l'utilisation par la culture des engrais est très différente; le coefficient réel d'utilisation est de $14 \mathrm{p} .100$ pour le DAP, de 2 p. 100 pour le phospal et 0,9 p. 100 pour le phosphate naturel. Ces résultats permettent alors de calculer, même en l'absence de réponse culturale, une efficacité relative du phospal et du phosphate naturel, par rapport au phosphate d'ammonium, définie par le rapport de leur coefficient réel d'utilisation. Elle a pour valeur $16 \mathrm{p} .100$ pour le phospal et 7 p. 100 pour le phosphate naturel. Ces résultats permettent également d'expliquer pourquoi les engrais phosphatés, avec les méthodes culturales en vigueur, ne provoquent de réponse culturale positive que dans les sols pauvres à très pauvres. Un supplément de prélèvement en phosphore compris entre 1 et 15 p. 100 de la quantité apportée comme engrais ne sera significatif que si la quantité de phosphore présente dans la plante et originaire du sol est très faible c'est-à-dire si l'on est en sol insuffisamment pourvu. Cette analyse est largement confirmée par l'examen des données bibliographiques qui montre que les sols présentant une réponse au phospal, sont toujours très pauvres en $\mathrm{P}$ assimilable, leur teneur étant généralement inférieure à 10 p.p.m.POLSEN. De plus, dans ces circonstances, l'efficacité immédiate du phosphal n'atteindra que 38 p. 100 de celle de la forme soluble.

Cet ensemble de résultats expérimentaux et bibliographiques semble en contradiction avec quelques conclusions, antérieurement publiées, faisant état d'une équivalence de comportement du phospal et du superphosphate. Pour ce qui concerne l'effet à court terme, nous pouvons affirmer qu'il n'y a pas réellement contradiction, dans la mesure où l'équivalence de comportement du phospal et du superphosphate s'observe essentiellement dans des circonstances agronomiques où les critères classiques (rendement, prélèvement de phosphore) ne peuvent révéler l'utilisation effective du phosphore des différentes formes d'engrais. Pour ce qui concerne l'effet résiduel, une analyse systématique des essais de longue durée, ayant reçu du phosphate alumino-calcique apporterait un important complément d'informations.

Recu le 25 septembre 1986 Accepté le 14 septembre 1987.

\section{RÉFÉRENCES BIBLIOGRAPHIQUES}

Afnor, 1982. Matières fertilisantes et supports de cultures. Recueil de normes françaises. Afnor, Paris, $1^{\text {re }}$ éd., 409 p.

Arvieu J. C., 1980. Réactions des phosphates minéraux en milieu calcaire ; conséquences sur l'état et la solubilité du phosphore. Bull. Assoc. fr. Etude Sol, 3, 179-189.

Barbier G., Tyskiewicz E., 1952. Mobilité des ions phosphoriques fixes dans le sol étudiée au moyen de 32P. A.I.S.S. Commissions $I I$ et $I V, 79-81$.
Black C. A., Scott C. O., 1956. Fertilizer evaluation. 1. Fundamental principles. Soil Sci. Soc. Am. Proc., 20, 176-179.

Blanchet R., Bosc M., Gelfi, Hilaire A., 1971. Vieillissement d'engrais phosphatés dans le sol et conduite de la fertilisation. Ann. agron., 22, 687-703

Bolland M. D. A., Bowden J. W., 1982. Long term availability of phosphorus from calcined rock phosphate compared with superphosphate. Aust. J. agri. Res., 33, 1061-1071. 
Bolland M. D. A., Bowden J. W., 1984. The initial and residual value for subterranean clover of phosphorus from crandallite rock phosphate, apatite rock and superphosphate. Fertil. Res., 5, 295 307.

Bolland M. D. A., Bowden J. W., D'Antuono H. F., Gilkes R. J., 1984. The current and residual value of superphosphate, Christmas island C-grade ore and Calciphos for a subterranean clover pasture. Fertil. Res., 5, 335-354.

Bolland M. D. A., Weatherley A. J., Gilkes R. J., Bowden J. W., 1986. Granular reactive apatite rock phosphate is not an effective phosphorus fertilizer in the short term on lateritic soils in southwestern Australia. Aust. J. exp. Agric., 26, 217-225.

Boniface R., Fardeau J. C., Trocme S., 1975. Observations sur les fumures phosphatées d'entretien. C.R. Acad. Sci., Ser. D: Sci. nat., 173-183.

Buron P., 1965. Travaux des stations (Blois), 1961 et 1962. Dossier C.E.E. Essais agronomiques concernant le Phospal. Groupe de travail « Entraves aux échanges résultant de dispositions d'ordre technique »; engrais. Délégation française. p. 10.

Collier D., Gachon L., 1965. Travaux des stations (ClermontFerrand, 1963). Dossier C.E.E. Essais agronomiques concernant le Phospal. Groupe de travail « Entraves aux échanges résultant de dispositions d'ordre technique »; engrais. Délégation française. p. 17.

Cooke G. W., 1967. The control of soil fertility. Crosby Lockwood, Londres, $1^{\text {re }}$ éd., $526 \mathrm{p}$.

Coppenet M., Duval L., 1965. Travaux des stations (Quimper 1956 à 1960). Dossier C.E.E. Essais agronomiques concernant le Phospal. Groupe de travail «Entraves aux échanges résultant de dispositions d'ordre technique " ; engrais. Délégation française. p. 9.

Couey M., Bouyer S., Chabrolin R., Courtessole F., 1968. Réponse du riz à la fumure dans la région du fleuve Sénégal, p. 720-730. In Colloque sur la fertilisation dans les sols tropicaux, t. 1, IRAT, Paris, $1254 \mathrm{p}$.

Depardon L., Buron P., 1953. Effets comparés de l'acide phosphorique sous diverses formes sur blé. Ann. agron., 6, 926-927.

Depardon L., Buron P., 1954. Effets comparés de l'acide phosphorique sous diverses fromes sur Avoine. Ann. agron., 5, 815-816.

Depardon L., Buron P., 1955. Effets comparés de l'acide phosphorique sous diverses formes sur betteraves $1 / 2$ sucrières. Ann. agron., $5,822-823$.

Depardon L., Buron P., 1956. Effets comparés de l'acide phosphorique sous diverses formes sur blé d'automne. Ann. agron., 7, 996997.

Depardon L., Buron P., 1957. Effets comparés de l'acide phosphorique sous diverses formes sur betteraves fourragères. Ann. agron., 5, $711-712$.

Depardon L., Buron P., 1958. Effets comparés de l'acide phosphorique sous diverses formes sur blé en Beauce. Ann. agron., 3, 388 389.

Fardeau J. C., Jahiel M., Morel C., Jappe J., 1984. How to fight the fixing of phosphatic fertilizer in the soil: isotopic results, p. 327-331. In : E. Welte \& I. Szabolcs. Fight against hunger through improved plant nutrition, vol. 2. C.I.E.C., Goettingen. $505 \mathrm{p}$.

Flicoteaux R., 1982. Genèse des phosphates alumineux du Sénégal occidental. Etapes et guides de l'altération. Mémoire $n^{\circ} 67$ CNRS, Université Louis Pasteur. 245 p.

Fried M., Dean L. A., 1952. A concept concerning the measurement of available soil nutrients. Soil Sci., 73, 263-271.

Gachon L., 1965. Travaux des stations (Clermont-Ferrand, 1963). Dossier C.E.E. Essais agronomiques concernant le Phospal. Groupe de travail « Entraves aux échanges résultant de dispositions d'ordre technique " ; engrais. Délégation française. p. 18.

Gachon L., 1973. Vieillissement de divers engrais phosphatés en relation avec le type de sol, étudié par la méthode Chang et Jackson. Ann. agron., 24 (5), 585-613.
Gachon L., 1977. Utilité d'un bon niveau de réserves phosphatées du sol. Phosphore Agric., 70, 27-33.

Garola J., 1956. Comparaison de divers engrais phosphatés. Ann. agron., 6, 995-996

Gros A., 1962. Engrais. Guide pratique de la fertilisation. La Maison Rustique, Paris, $3^{\mathrm{e}}$ éd., 442 p.

Hebert J., 1965. Travaux des stations (Laon, 1956 à 1964). Dossier C.E.E. Essais agronomiques concernant le Phospal. Groupe de travail « Entraves aux échanges résultant de dispositions d'ordre technique " ; engrais. Délégation française. p. 21.

Hebert J., Rémy J. C., 1965. Travaux des stations (Laon, 1956 à 1963). Dossier C.E.E. Essais agronomiques concernant le Phospal. Groupe de travail « Entraves aux échanges résultant de dispositions d'ordre technique »; engrais. Délégation française. p. 20.

Jahiel M., Morel Ch., 1983. Analyse du devenir de différents phos phates dans plusieurs sols de $\mathrm{pH}$ variant de 5,2 à 6,6. Mémoire de fin d'études. I.S.A. Lille, 119 p.

Lefevre G., Hiroux G., 1965. Travaux des stations (Amiens, 1961 à 1963). Dossier C.E.E. Essais agronomiques concernant le Phospal. Groupe de travail « Entraves aux échanges résultant de dispositions d'ordre technique »; engrais. Délégation française. 13-15.

Leon L. A., Fenster W. E., Hammond L. L., 1986. Agronomic potential of eleven phosphate rocks from Brasil, Colombia, Peru and Venezuela. Soil Sci. Soc. Am. J., 50, 798-802.

Lipsett J., Williams C. H., 1970. Evaluation of Christmas Island C grade phosphate as a fertilizer on some soils in Southern New South Wales. Austr. J. exp. Agri. anim. Husb., 10, 783-789.

Loue A., 1982. Comparaison entre le blocage de la fumure potassique en tête de rotation et sa répartition sur les cultures. Dossier K20. SCPA 18, 1-23.

Malterre H., Fioramonti S., 1957. Fumure phosphatée des blés d'automne dans la région de Toulouse. Ann. agron., 5, 708-711.

Mason M. G., Cox W. J., 1969. Calcined rock phosphate as fertilizer for pasture and cereal production in Western Australia. Austr. $J$ exp. Agri. anim. Husb., 9, 99-104.

Moriceau L., Tintignac J. P., 1963. Comportement du phospal en sol calcaire et en sol acide. C.R. Acad. Agric. Fr., 1351-1359.

Muller F. B., 1970. Agronomic use of calcined Christmas Island iron/aluminium phosphates. 1. Field trials. N. Z. J. agric. Res., 13, 453-464.

Palmer B., Bolland M. D. A., Gilkes R. J., 1979. A reevaluation of the effectiveness of calcined Christmas Island C-grade rock phosphate. Aust. J. exp. Agric. anim. Husb., 19, 605-610.

Radet E., 1958. Evolution de phosphates hyposolubles dans les sols de craie. Bull. Assoc. fr. Etude sol, Sci. sol, 3, 102-108.

Radet E., 1962. Contribution à l'étude du comportement des engrais phosphatés dans les sols de craie. Bull. Assoc. fr. Etude Sol, Sci. sol, 5, 287-305.

Roberts O. T., White M. S., 1974. Some factors affecting agronomic response to apatites and to calcined Christmas Island iron/ aluminium phosphates. N. Z. J. agric. Res., 17, 479-484.

Singh M., 1983. Study on Senegalese calcined phosphate rock and single superphosphate in rice and wheat rotation, p. 543-548. In : Actes du $3^{e}$ congrès international sur les composés phosphorés. IMPHOS, Casablanca, $656 \mathrm{p}$.

Teitzel J. K., Bruce R. C., 1969. Relative efficiency of two sources of phosphorus in the establishment of tropical pasture species. Queensl. J. agric. anim. Sci., 26, 235-242.

Tendille, 1954. Essai du phospal en vase de végétation sur sol brun calcaire. Ann. agron., 5, 813-814.

Truong Binh, Chevassus-Rosset C., Djegui N., 1984. Etude de l'évolution de trois phosphates (DAP, TSP, Phospal) dans un sol sableux calcaire du désert de Lybie. Agron. trop., Paris, 39, 31-33.

White R. F., Kempthorne O., Black C. A., Webb J. R., 1956. Fertilizer evaluation. 2. Estimation of availability coefficients. Soil Sci. Soc. Am. Proc., 20, 179-183. 